\title{
The Biodiversity of Marine Gastropods of Thailand in the Late Decade.
}

\author{
Kitithorn Sanpanich $^{{ }^{*}}$ and Teerapong Duangdee ${ }^{2}$
}

${ }^{1}$ Institute of Marine Science, Burapha University, Tambon Saensook, Amphur Moengchonburi, Chonburi, 20131 Thailand. ${ }^{2}$ Department of Marine Science, Faculty of Fisheries, Kasetsart University, Bangkok, 10900 Thailand.

*kitithor@buu.ac.th (Corresponding author)

\begin{abstract}
This study is mainly based on the collection of marine gastropods along the east coast of the Gulf of Thailand which had been carried out along the coastline in 55 sites from the province of Chonburi to Trad during April 2005 - December 2009. As many habitats as possible were examined at each sites from sandy beaches, muddy sand, rocky shore, and coral reefs. A total of 306 species of gastropods were collected and had been classified in53families 116genera.The most widespread species were Planaxis sulcatus (Planaxidae) and Polinices mammilla (Naticidae) found in 37 sites, followed by Echinolittorina malaccana (Littorinidae) in 35 sites. The highest diversity was 187 species in Trat whereas Koh Mark had the most abundance in this area. The lowest diversity was in Chanthaburi, 88 species, whereas Koh Nomsoa was the most abundant site.The diversity of gastropods in Chonburi was 152 species, whereas Koh Samaesarn and Koh Juang were the most abundant site. 137 species had been found in Rayong and Koh Munnai was the most abundant site. The data from this study had been compared with the resent studies in the late decade from the west coast of the Gulf of Thailand and Andaman Sea.The total gastropods in the late decade were 454 species 205 genera 69 families.
\end{abstract}

(Keywords: marine gastropods, distribution, diversity, Gulf of Thailand)

\section{INTRODUCTION}

Thailand has the total sea areas about 350,000 square kilometers. The long coast line of about 2,815 km (measured along the shore line) and more than 900 islands. There are two coast lines geographically from border of Trat province to Su Ngai Ko Lok Estuary, Narathiwat province in the Gulf of Thailand and the another coast line in Andaman Sea from the edge of Ranong province to theborder of Malaysia at Satun province.Since, Suvatti reported the checklists of marine gastropods all over the country about 60 species in more than 70 years ago $[1,2]$, there were very few people studied on these animals in term of diversity. Tanasiriwong reported 248 species from Phuket [3], Bussarawit reported 165 species from Surin and LePae Island [4] and Swennen et al. reported 117 species from Pattani and Narathiwat provinces [5].Chon Buri, Rayong, Chathaburi and Trat provinces on the east coast of the Gulf are the attractive areas for marine mollusks diversity because there are a lot of habitats suitable for them to survive such as many beaches and islands. This study aims to collect marine gastropods mainly from the east coast and report the species lists of the mollusks in the late decade.

\section{MATERIALS AND METHODS}

The present specimens of marine gastropods were collected from sandy beaches, muddy sand, rocky shores, and coral reefs at islands along the Chonburi to Trad coastline fromApril 2005 - December 2009. The total of 55 survey sites (Figure 1; Table1), there were 20 sites in Chonburi, 10 sites in Rayong, 7 sites in Chanthaburi and 18 sites in Trad. The study was conducted by using SCUBA diving randomly in daytime from shallow water on the reef flat to the reef slope at the maximum depth of about $18 \mathrm{~m}$ around all islands and by hand-picking along the beach and rocky shore to obtain as many species as possible. Both living and dead shells were collected whereas the dead shells will represent species living at the site. All specimens were recorded and identified, the living specimens were preserved in $10 \%$ formalin for about 3 days then moved to $70 \%$ alcohol. Shells were cleaned in sodium hypochloride $6.6 \% \mathrm{w} / \mathrm{v}$ overnight then washed by tap water and dried for further study. Length and width were measured using vernier calipers. The specimens are deposited in the reference collection museum of the Institute of Marine Science, Burapha University. The classification is based on Beesley et al [6]. The collected specimens 
were identified by compared with illustrations, general descriptions, and specific characters in the available literature (Abbott [7], Abbott and Dance[8], Eisenberg [9], Habe [10], Kira [11], Nielsen [12], Okutani [13], Swennen et al [5], Tan and Chou [14], Wilson [15] and Wilson [16],Hylleberg and Kilburn [23], Bouchet et al [24], Boxshall et al [25]).

\section{RESULTS}

A total of 306 species of marine gastropods along the east coast of the Gulf of Thailand from 55 sites were recorded and had been identified in 53 families 116genera (Table 2). Planaxis sulcatus (Planaxidae) and Polinicesmammilla (Naticidae) found in 37 sites, followed by Echinolittorina malaccana (Littorinidae) in 35 sites. There were 1319individual occurrencesfound in the 55 sites, giving a mean of $24.0 \pm 0.8$ individual occurrences per site; the range was from 5 to 65 species (Table 1). The highest diversity was 186 species in Trad whereas Mark Island had the most abundance in this area. The lowest diversity was in Chanthaburi, 92 species, whereas Kungwiman was the most abundant site.The diversity of gastropods in Chonburi was 151 species, whereas Samaesarn and Juang Islandwere the most abundant site. 140 species had been found in Rayong and Munnai Island was the most abundant site. The lowest diversity was registered in the Chanthaburi province (sites 32, 34, 35), 87 species, with Nerita albicilla (Neritidae) and Turbo bruneus (Turbinidae) being the most common species, within this province, Kungwiman was the site that presented the highest number of species. The highest diversity was found in Trad province with 186 species, being Polinicesmammilla (Naticidae) the most common species as same as in Chonburi. Of the 10 sites sampled in Rayong, Munnai Island was the one that presented a higher number of species. In the Chonburi province there were identified 151 species.Sites with both the largest and smallest recorded gastropod diversity varied geographically (Tables 1and 2).

\section{DISCUSSION}

Since, this study had reported a total of 306 species of marine gastropods mainly just along the east coast of the Gulf of Thailand from 55 sites in 4 provinces. However, the secondary data from many reports in the late decade of the country also be compared and made the total species lists as 454 species not included nudibranches. Family Calliostomatidae,Personidae, Tonnidae, Velutinidae, Colubrariidae, Eulimidae,Epitoniidae and Bullinidae occurred only in the Andaman Sea whereas familyChilodontidae, Turridae, Xenophoridae, Cancellariidae, Drilliidae, Clavatulidae, Bursidae, Acteonidae and Amathinidae reported just only in the Gulf of Thailand. The distribution of gastropods along the eastern coast is widely spread not specific in any species except in some species which found only in such one site (Table 2) but it does not mean that there is no chance to find in the other places. However, this study will be useful to everybody who needs to look for such the specific species or family of gastropods, they can easily follow the places in the checklist sites. Lastly, this study adds to the database on the distribution of marine gastropods of the country along the east coast of the Gulf of Thailand.

\section{ACKNOWLEDGEMENTS}

I would like to thank for the staff of biodiversity research unit of the Institute of Marine Science, Burapha University and Department of Marine Science, Faculty of Fisheries, Kasetsart University for collecting specimens.

\section{REFERENCES}

1. Suvatti, C. (1937). A check-list of aquatic fauna in Siam (excluding fishes). Bureau of Fisheries, Bangkok.

2. Suvatti, C. (1938). Molluscs of Siam. Bureau of Fisheries, Bangkok.

3. Tanasiriwong, R. (1978). An Illustrated checklist of marine shelled gastropods from Phuket Island, Adjacent mainland and offshore island, Western Peninsular Thailand. Phuket Marine Biological Center Research Bulletin. 21:1-63

4. Bussarawit,S. (1995). Molluscs from the marine national parks: Surin and Le-Pae Island, Andaman Sea, Thailand. Phuket Marine Biological Center Special Publication. 15: 119-126

5. Swennen C., R. G. Moolenbeek, N. Ruttanadakul, H. Hobbelink, H. Dekker, and S. Hajisamae. 2001. The Molluscs of the Southern Gulf of Thailand . Thai Studies inBiodiversity No. 4.

6. $\quad$ Beesley PL, Ross GJB and Wells A (eds) (1998) Molluscs: The Southern Synthesis. Fauna of Australia, Volume 5, CSIRO Publishing, Melbourne.

7. Abbott, R.T. (1991). Seashells of Southeast Asia. Tynron Press, Thornhill. 
8. Abbott,R.T. and Dance,S.P. (1990). Compendium of Seashells. American Malacologists, Inc., Florida.

9. Eisenberg, J.M. (1989) A Collector's Guide to Seashells of the World Crescent Books, New York.

10. Habe, T. (1975) Shells of the Western Pacific in Color Vol.II, Hoikusha Publishing Co., Ltd., Osaka.

11. Kira, T. (1975) Shells of the Western Pacific in Color Vol.I, Hoikusha Publishing Co., Ltd., Osaka.

12. Nielsen, C. (1976) An Illustrated Checklist of Gastropods from PMBC Beach with a Reef-flat at Bhuket, Thailand. Phuket Marine Biological Center Research Bulletin. 9:1-26

13. Okutani, T. (2000) Marine Mollusks in Japan Tokai University Press, Tokyo.

14. Tan, K.S. and Chou, L.M. (2000) A guide to common seashells of Singapore Singapore Science Center.

15. Wilson, B., (1993). Australian Marine Shells 1. Odyssey Publishing, Kallaroo.

16. Wilson, B., (1994). Australian Marine Shells 2. Odyssey Publishing, Kallaroo.

17. Duangdee, T., Sanpanich, K. and Thongpinyochai, S. (2011). The species diversity of marine mollusks in coral reefs at Ang Thong Islands, Surat Thani Province. Proceeding of $5^{\text {th }}$ RSPG Researchers Club Conference "Step to The World Wide with Confidence", $3^{\text {rd }}-5^{\text {th }}$ November 2011, Nong Rawieng Training Center, Rajamangala University of Technology Isan, Nakhon Ratchasima Province. p.235-250.

18. Department of Marine Science. (2010). Project on Biodiversity Inventory and Information Management in the Biodiversity Hotspot of Ranong, Phangnga, Krabi and Phuket Provinces. Final report present to Office of Natural Resources and Environmental Policy and Planning. Faculty ofFisheries, Kasetsart University. Bangkok.

19. Forestry Research Center. (2006). Biodiversity of Marine \& CoastalEcosystem. Biodiversity Survey \& Information System Project. Volume 3. Final report present to Office of Natural Resources andEnvironmental Policy and Planning. Faculty of Forestry, KasetsartUniversity. Bangkok.

20. Duangdee, T., Sanpanich, K. and Thongpinyochai, S. (2009). The species diversity of marine mollusks in coral reefs and adjacent area at Tarutoa National Park, Satun Province. Proceeding of $4^{\text {th }}$ RSPG Researchers Club Conference "Thai Resources: Turn to The New Way in Thai Way (Thai Innovation)”, $20^{\text {th }}-23^{\text {rd }}$ October 2009, Khao
Kheow Open Zoo, Si Racha, Chon Buri Province. p.251-266.

21. Duangdee, T. (2004).Biodiversity of mollusks of Chang Islands in: Final Report. Research Program on Aquatic Resources and Environment Potentials for Development of Eco-tourism of Chang Islands.p.1-39

22. Sanpanich, K., Wells, F.E. and Chitramvong, Y. (2004). Distribution of the family Littorinidae (Mollusca: Gastropoda) in Thailand. Records of the Western Australian Museum 22: 241-251.

23. Hylleberg, J. and Kilburn, R.N. (2003). Marine Molluscs of Vietnam. Phuket Marine Biological Center Special Publication. 28: 1-300

24. Bouchet, P., Rocroi, J.P., Fryda, J., Hausdorf, B., Ponder, W., Valdes, A. and Waren, A. (2005). Classification and nomenclator of gastropod families. Malacologia. 47 (1-2): 1-397.

25. Boxshall, G., Mees, J., Costello, M. J., Hernandez, F., Vandepitte, L., Gofas, S., Hoeksema, B.W., Klautau, M., Kroh, A., Poore, G.C.B., Read, G., Stöhr, S., de Voogd, N.J., Walter, C.T., De Broyer, C., Horton, T. and Kennedy, M. (eds) (2013). World Register of Marine Species. Accessed at http://www.marinespecies.org on 2013-08-1. 


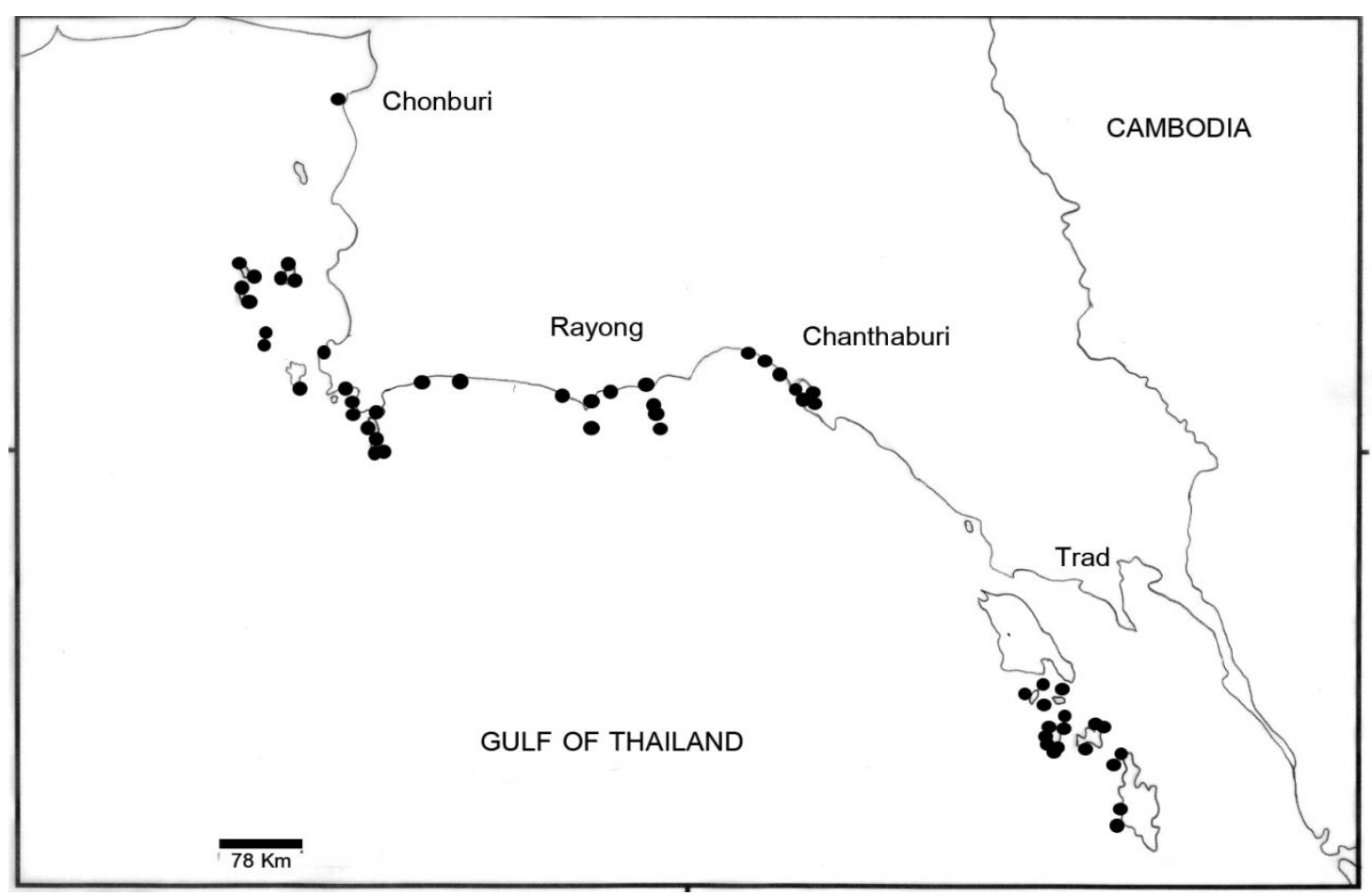

Figure 1. Collection Sites (dots) along the east coast of the Gulf of Thailand

Table 1. Summary of collection sites and total number of bivalve species collected along the eastern coast of the Gulf of Thailand. ( $\mathrm{S}$ = sandy beach; $\mathrm{R}$ = rocky shore; $\mathrm{C}=$ coral reef; $\mathrm{M}=$ muddy sand)

\begin{tabular}{|c|c|c|c|c|}
\hline Site no. & Site name & Habitats & Coordinates & $\begin{array}{l}\text { Number of } \\
\text { species }\end{array}$ \\
\hline 1 & Angsila, Chonburi & $\mathrm{S}, \mathrm{M}$ & $13^{\circ} 18 ’ 57.56 ” \mathrm{~N} ; 100^{\circ} 55^{\prime} 4.44^{\prime} \mathrm{E}$ & 10 \\
\hline 2 & Larn Island, Pattaya, Chonburi & $\mathrm{C}$ & 1255’39.30”N; 10046’29.60”E & 12 \\
\hline 3 & Krok Island, Pattaya, Chonburi & $\mathrm{C}$ & $12^{\circ} 55^{\prime} 42.299^{\prime \prime}$; 10048’16.31’'E & 11 \\
\hline 4 & Sark Island, Pattaya, Chonburi & $\mathrm{C}$ & $12^{\circ} 56^{\prime} 42.15^{\prime \prime N}$; $100^{\circ} 47^{\prime} 30.30^{\prime} \mathrm{E}$ & 10 \\
\hline 5 & Loerm Island, Pattaya, Chonburi & $\mathrm{C}$ & 1257’36.39’N; 100³9’13.11’E & 14 \\
\hline 6 & Pai Island, Pattaya, Chonburi & $\mathrm{C}$ & $12^{\circ} 56^{\prime} 35.52^{\prime \prime N}$; 10040’38.52”E & 21 \\
\hline 7 & Klungbadarn Island, Pattaya, Chonburi & $\mathrm{C}$ & $12^{\circ} 54^{\prime} 12.78^{\prime \prime} \mathrm{N}$; 10040’46.69”E & 14 \\
\hline 8 & Marnvichai Island, Pattaya, Chonburi & $\mathrm{C}$ & $12^{\circ} 52^{\prime} 30.79 ” \mathrm{~N} ; 100^{\circ} 40^{\prime} 27.42^{\prime \prime} \mathrm{E}$ & 18 \\
\hline 9 & Rin Island, Pattaya, Chonburi & $\mathrm{C}$ & $12^{\circ} 47^{\prime} 43.61 ” \mathrm{~N} ; 100^{\circ} 42^{\prime} 17.08^{\prime \prime} \mathrm{E}$ & 31 \\
\hline 10 & Hin Hoo-chang, Pattaya, Chonburi & $\mathrm{C}$ & $12^{\circ} 48^{\prime} 34.55^{\prime \prime N}$; $100^{\circ} 42^{\prime} 31.45^{\prime} \mathrm{E}$ & 15 \\
\hline 11 & Sor Beach, Toong Prong, Sattahip, Chonburi & $\mathrm{R}, \mathrm{S}$ & $12^{\circ} 43^{\prime} 54.9 ’ \mathrm{~N} ; 100^{\circ} 50^{\prime} 02.2^{\prime \prime} \mathrm{E}$ & 9 \\
\hline 12 & Nang-rong Beach, Sattahip, Chonburi & S, C & 12³6’55.87’N; 10055’13.64”E & 24 \\
\hline 13 & Jorrake Island, Sattahip, Chonburi & $\mathrm{C}$ & $12^{\circ} 36^{\prime} 10.19 ” \mathrm{~N} ; 100^{\circ} 55^{\prime} 4.13 ” \mathrm{E}$ & 38 \\
\hline 14 & Sattahip Bay, Sattahip, Chonburi & S, M & $12^{\circ} 39^{\prime} 37^{\prime \prime N}$; $100^{\circ} 53^{\prime} 45.8^{\prime \prime} \mathrm{E}$ & 23 \\
\hline 15 & Kram Island, Sattahip, Chonburi & S, C & $12^{\circ} 40^{\prime} 19.2^{\prime \prime} \mathrm{N} ; 100^{\circ} 47^{\prime} 10.3 ” \mathrm{E}$ & 34 \\
\hline 16 & Chong Samaesarn, Sattahip, Chonburi & $\mathrm{C}$ & $12^{\circ} 35^{\prime} 46.92^{\prime \prime N}$; $100^{\circ} 57^{\prime} 47.37 ” \mathrm{E}$ & 18 \\
\hline 17 & Samaesarn Island, Sattahip, Chonburi & S, C & 12³3’58.72”N; 10056’57.95”E & 53 \\
\hline
\end{tabular}


Table 1. (Cont.)

\begin{tabular}{|c|c|c|c|c|}
\hline Site no. & Site name & Habitats & Coordinates & $\begin{array}{l}\text { Number } \\
\text { of } \\
\text { species }\end{array}$ \\
\hline 18 & Karm Island, Sattahip, Chonburi & $\mathrm{C}$ & 12³4’27.93”N; 10055’59.12”E & 27 \\
\hline 19 & Juang Island, Sattahip, Chonburi & $\mathrm{C}$ & $12^{\circ} 30^{\prime} 50.67 ’ \mathrm{~N} ; 100^{\circ} 57^{\prime} 12.93 ” \mathrm{E}$ & 53 \\
\hline 20 & Jarn Island, Sattahip, Chonburi & $\mathrm{C}$ & 12³1’2.19”N; 10058’16.74”E & 49 \\
\hline 21 & Sai-thong Beach, Moeng, Rayong & $\mathrm{S}$ & 1240’11.04”N; 101¹1'11.81’'E & 19 \\
\hline 22 & Numrin Beach, Ban Chang, Rayong & $\mathrm{S}$ & $12^{\circ} 40^{\prime} 31.25 ” \mathrm{~N} ; 101^{\circ}$ 5’31.90”E & 12 \\
\hline 23 & Maerumpung Beach, Klaeng, Rayong & $\mathrm{S}$ & 12³7'19.43’'N; 101²1’48.16’"E & 16 \\
\hline 24 & Ban Pe, Klaeng, Rayong & $\mathrm{S}$ & $12^{\circ} 36^{\prime} 43.87^{\prime \prime} \mathrm{N} ; 1^{\circ}{ }^{\circ} 25^{\prime} 22.18^{\prime \prime} \mathrm{E}$ & 12 \\
\hline 25 & Suanson Beach, Klaeng, Rayong & $\mathrm{S}$ & $12^{\circ} 37^{\prime} 55.08^{\prime \prime} \mathrm{N} ; 1^{\circ} 21^{\circ} 27.16^{\prime} \mathrm{E}$ & 17 \\
\hline 26 & Samed Island, Klaeng, Rayong & $\mathrm{R}, \mathrm{C}$ & 12³4’0.07’N; 101²7’51.37’E & 33 \\
\hline 27 & Munnai Island, Klaeng, Rayong & S, C & 12³6’51.23”N; 10144'13.54”E & 55 \\
\hline 28 & Munklarng Island, Klaeng, Rayong & S, C & 12³5’59.16”N; 10144'37.31’'E & 32 \\
\hline 29 & Munnork Island, Klaeng, Rayong & S, C & 12³4’9.52”N; 10142’8.60”E & 54 \\
\hline 30 & Laem Maepim, Klaeng, Rayong & $\mathrm{S}$ & 12³8’37.14”N; 101³8’8.83”E & 8 \\
\hline 31 & Kungwimarn, Chanthaburi & $\mathrm{R}, \mathrm{C}$ & 12³6’30.16”N; 10152’11.99”E & 40 \\
\hline 32 & Kung Kraben, Chanthaburi & M & 12³4'26.58’'N; 10153'47.37’'E & 8 \\
\hline 33 & Joaloa Beach, Chanthaburi & S, C & 12³2’4.68”N; 10156’3.95”E & 10 \\
\hline 34 & Moo Bay, Chanthaburi & $\mathrm{S}, \mathrm{M}$ & $12^{\circ} 29^{\prime} 01.83^{\prime \prime} \mathrm{N} ; 102^{\circ} 02^{\prime} 38.33^{\prime \prime} \mathrm{E}$ & 5 \\
\hline 35 & Yang Bay, Chanthaburi & S, R & $12^{\circ} 28^{\prime} 31.50 ” \mathrm{~N} ; 102^{\circ} 2^{\prime} 40.16^{\prime \prime} \mathrm{E}$ & 8 \\
\hline 36 & Saba Island, Chanthaburi & $\mathrm{C}$ & 12³0’33.33’N; 10156’56.59’'E & 17 \\
\hline 37 & Nomsoa Island, Chanthaburi & $\mathrm{C}$ & $12^{\circ} 27^{\prime} 53.50^{\prime \prime} \mathrm{N} ; 102^{\circ} 01^{\prime} 24.41^{\prime \prime} \mathrm{E}$ & 38 \\
\hline 38 & Loaya Island, Trat & $\mathrm{C}$ & $11^{\circ} 56^{\prime} 43.7^{\prime \prime} \mathrm{N} ; 102^{\circ} 24^{\prime} 04.1^{\prime \prime} \mathrm{E}$ & 24 \\
\hline 39 & Wai Island, Trat & $\mathrm{C}$ & 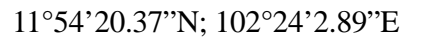 & 56 \\
\hline 40 & Ngarm Island, Trat & $\mathrm{C}$ & $11^{\circ} 56^{\prime} 54.88^{\prime \prime} \mathrm{N} ; 102^{\circ} 26^{\prime} 15.77^{\prime \prime} \mathrm{E}$ & 9 \\
\hline 41 & Kloom Island, Trat & $\mathrm{C}$ & $11^{\circ} 55^{\prime} 22.08^{\prime \prime} \mathrm{N} ; 102^{\circ} 21^{\prime} 12.20^{\prime \prime} \mathrm{E}$ & 35 \\
\hline 42 & Kumpun Island, Trat & $\mathrm{R}, \mathrm{C}$ & 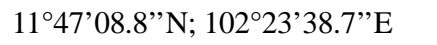 & 12 \\
\hline 43 & Mapring Island, Trat & $\mathrm{R}, \mathrm{C}$ & 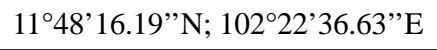 & 24 \\
\hline 44 & Songpeenong Island, Trat & $\mathrm{C}$ & $11^{\circ} 48^{\prime} 32.7^{\prime \prime} \mathrm{N} ; 102^{\circ} 22^{\prime} 43.5^{\prime \prime} \mathrm{E}$ & 15 \\
\hline 45 & Hin Yak Island, Trat & $\mathrm{C}$ & $11^{\circ} 47^{\prime} 19.67^{\prime \prime} \mathrm{N} ; 102^{\circ} 23^{\prime} 42.39^{\prime \prime} \mathrm{E}$ & 8 \\
\hline 46 & Rung Island, Trat & $\mathrm{C}$ & $11^{\circ} 47^{\prime} 46.32^{\prime \prime} \mathrm{N} ; 102^{\circ} 22^{\prime} 56.25^{\prime \prime} \mathrm{E}$ & 28 \\
\hline 47 & Hin Plai num, Trat & $\mathrm{C}$ & $11^{\circ} 51^{\prime} 03.1^{\prime \prime} \mathrm{N} ; 102^{\circ} 24^{\prime} 42.0^{\prime \prime} \mathrm{E}$ & 9 \\
\hline 48 & Thonglarng Island, Trat & $\mathrm{C}$ & $11^{\circ} 49^{\prime} 11.47^{\prime \prime N}$; 102²4’4.00”E & 13 \\
\hline 49 & Rayungnok Island, Trat & $\mathrm{C}$ & $11^{\circ} 48^{\prime} 0.23 ” \mathrm{~N} ; 102^{\circ} 27^{\prime} 8.64^{\prime \prime} \mathrm{E}$ & 7 \\
\hline 50 & Kradad Island, Trat & $\mathrm{C}$ & 1151'1.03”N; 102³0’55.72”E & 20 \\
\hline 51 & Mark Island, Trat & $\mathrm{C}$ & 1150’34.79”N; 102²9’35.09”'E & 65 \\
\hline 52 & Maisee Island, Trat & $\mathrm{C}$ & $11^{\circ} 43^{\prime} 17.38^{\prime \prime} \mathrm{N}$; 102³0’41.52”E & 50 \\
\hline 53 & Hin Bungbao, Trat & $\mathrm{C}$ & 11³5’50.66”N; 102³1’46.38”'E & 14 \\
\hline 54 & Pukwaen Bay, Trat & $\mathrm{C}$ & 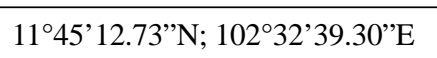 & 13 \\
\hline 55 & Hin Kong Bay, Trat & $\mathrm{C}$ & 11³7’29.85”N; 102³2’31.69”E & 49 \\
\hline
\end{tabular}


Table 2. List of gastropods collected at sites along the east coast of the Gulf of Thailand. A = record in Andaman Sea; $\mathrm{W}=$ record in west of the Gulf of Thailand; Duangdee et al. [17]; Department of Marine Science[18]; Forestry Research Center[19]; Duangdee et al. [20]; Duangdee [21]; Sanpanich et al.[22]

\begin{tabular}{|c|c|}
\hline Family Lottidae & \\
\hline Nipponacmea nigrans (Kira, 1961) & 20 \\
\hline Patelloidapygmaea(Dunker, 1860) & $20,39,46$ \\
\hline Patelloida saccharina (Linnaeus,1758) & 6,10,17,19,20,31,A[18],[19],[20] \\
\hline Patelloida striataQouy \& Gaimard, 1834 & $\mathrm{~W}[17]$ \\
\hline \multicolumn{2}{|l|}{ Family Nacellidae } \\
\hline Cellana conciliata Iredale, 1940 & 20 \\
\hline Cellana grata (Gould, 1859) & {$[21], \mathrm{A}[20]$} \\
\hline Cellana radiata (Born,1778) & 19,31 \\
\hline Cellana rota (Gmelin, 1791) & $\mathrm{A}[20]$ \\
\hline Cellana testudinaria (Linnaeus,1758) & 12,46,51,55, W [17],A [18],[19],[21] \\
\hline \multicolumn{2}{|l|}{ Family Patellidae } \\
\hline Scutellastra barbara(Linnaeus,1758) & 29 \\
\hline Scutellastra flexuosa(Quoy \& Gaimard,1834) & 20, W[17] \\
\hline \multicolumn{2}{|l|}{ Family Haliotidae } \\
\hline HaliotisasininaLinnaeus,1758 & $17, \mathrm{~A}[18],[19]$ \\
\hline Haliotis ovina Gmelin,1791 & $6,8,12,13,15,17,19,20,29,41,42,45,51,[21], \mathrm{A}[19],[20]$ \\
\hline Haliotis planata (Sowerby, 1833) & $\mathrm{A}[18],[20]$ \\
\hline HaliotisvariaLinnaeus,1758 & 17,19,20,43,52,A[18],[20] \\
\hline \multicolumn{2}{|l|}{ Family Fissurellidae } \\
\hline Diodora rueppellii (G.B. Sowerby I,1835) & $3,4,17,25,27-29, W[17]$ \\
\hline Diodora ticaonica (Reeve, 1850) & $54,[21]$ \\
\hline Emarginula crassicostata (SowerbyII, 1863) & $\mathrm{A}[18],[19]$ \\
\hline Emarginella eximia (A. Adams, 1852) & $\mathrm{A}[18]$ \\
\hline Scutus sinensis (Blainville, 1825) & [17] \\
\hline Scutus unguis (Linneaus,1758) & 54 \\
\hline \multicolumn{2}{|l|}{ Family Calliostomatidae } \\
\hline Calliostoma iris Kuroda \& Habe, 1961 & $\mathrm{~A}[18]$ \\
\hline \multicolumn{2}{|l|}{ Family Chilodontidae } \\
\hline Euchelus asper(Gmelin, 1791) & 25 \\
\hline Herpetopoma aspersum (Philippi,1846) & 27 \\
\hline Herpetopoma atratum (Gmelin, 1791) & 21,27 \\
\hline Hybochelus cancellatus (Krauss, 1848) & 6 \\
\hline \multicolumn{2}{|l|}{ Family Trochidae } \\
\hline Chrysostoma paradoxum (Born,1778) & 5-10,15,16,19,20,29,43,51,52,55,[21],A[18],[20] \\
\hline Clanculus atropurpureus Gould,1849 & 39 \\
\hline Clanculus bicarinatus Angas, 1880 & 3 \\
\hline Clanculus comarilis Hedley, 1912 & 55 \\
\hline Clanculus consobrinus Tate, 1893 & 55 \\
\hline
\end{tabular}




\begin{tabular}{|c|c|}
\hline Clanculus denticulatus (Gray, 1827) & 16 \\
\hline Clanculus philippii (Koch, 1843) & 17 \\
\hline Clanculus margaritarius (Philippi,1846) & 15,19 \\
\hline Monilea belcheri (Philippi, 1849) & $27, \mathrm{~A}[20]$ \\
\hline Monilea callifera (Lamarck,1822) & $15,25,27,51,55, W[17]$ \\
\hline Monilea sp. & 55 \\
\hline Monodonta canalifera Lamarck, 1816 & [21] \\
\hline Monodonta labio (Linnaeus,1758) & 13,17,19,29,37,39,46,51,54,[21],W[17],A[18],[20] \\
\hline Pseudostomatella decolorata (Gould,1848) & 27 \\
\hline Stomatella impertusa (Burrows, 1815) & 19,27 \\
\hline Tectus pyramis (Born,1778) & 12,15,17-20,27,28,39,51,[21],A[18],[19],[20] \\
\hline Trochus nigropunctatus Reeve, 1861 & 9 \\
\hline Trochus histrio Reeve, 1848 & $\mathrm{~A}[19]$ \\
\hline Trochus maculatus Linnaeus, 1758 & $\begin{array}{l}\text { 7-9,13-15,17,19,20,25-29,37,39,46,51,52,54,[21],W[17] } \\
\text {,A[18],[19],[20] }\end{array}$ \\
\hline Tectus niloticus (Linnaeus, 1767) & 20,37,40,51,[21],W[17],A[18],[19],[20] \\
\hline Trochus radiatus Gmelin, 1791 & $\mathrm{~A}[18],[19],[20]$ \\
\hline Umbonium vestiarium (Linnaeus,1758) & $14,15,17,24-27,30,51,[21], \mathrm{A}[18],[19],[20]$ \\
\hline \multicolumn{2}{|l|}{ Family Angariidae } \\
\hline Angaria delphinus (Linnaeus,1758) & 6,8,12,13,15-17,19,20,26,29,51,[21],W[17],A[18],[19] \\
\hline \multicolumn{2}{|l|}{ Family Turbinidae } \\
\hline Astralium semicostatum (Kiener, 1850) & [21], A[18],[19],[20] \\
\hline Astralium haematragum(Menke, 1829) & $17, \mathrm{~A}[20]$ \\
\hline Astralium rhodostomum (Lamarck, 1822) & $13,15,17,19,20,39,43,45,48, A[18],[19],[20]$ \\
\hline Bolma henica (Watson, 1885) & $\mathrm{A}[18]$ \\
\hline Lunella cinerea (Born,1778) & [21] \\
\hline Turbo argyrostomus (Linnaeus, 1758) & [21],A[18],[19] \\
\hline Turbo bruneus (Roding,1798) & $\begin{array}{l}\text { 4,5,8,11-20,26-29,31,33,35-37,39,40,43,51-53,[21],W[17], } \\
\mathrm{A}[18],[19],[20]\end{array}$ \\
\hline Lunella coronata (Gmelin, 1791) & $\mathrm{A}[19]$ \\
\hline Turbo petholatus Linnaeus, 1758 & 5,8,9,15,17,19-21,42,43,45,[21], A[18],[19],[20] \\
\hline \multicolumn{2}{|l|}{ Family Neritidae } \\
\hline Nerita albicilla Linnaeus,1758 & $11,13,17,19,20,23,31,34-37,[21], \mathrm{W}[17], \mathrm{A}[18],[19],[20]$ \\
\hline Nerita balteataReeve,1855 & 13,19, A[18],[19] \\
\hline Nerita chamaeleon Linnaeus, 1758 & $17,20,29,37,[21], A[18],[19],[20]$ \\
\hline Nerita costata(Gmelin, 1791) & [21], A[18],[19],[20] \\
\hline Nerita planospiraAnton, 1839 & $\mathrm{~A}[20]$ \\
\hline Nerita polita Linnaeus,1758 & $19,29,37$ \\
\hline Nerita antiquata Recluz,1841 & 29 \\
\hline Nerita squamulata Le Guillou,1841 & 17,51, [21], A[19],[20] \\
\hline Nerita undata Linnaeus,1758 & $13,17,29,46,51,54,[21], A[18]$ \\
\hline Neritina violacea (Gmelin, 1791) & [21] \\
\hline Clithon oualaniense (Lesson,1831) & $1,11,14,16,32, \mathrm{~A}[20]$ \\
\hline
\end{tabular}




\begin{tabular}{|c|c|}
\hline Family Neritopsidae & \\
\hline Neritopsis radula (Linnaeus,1758) & 33,A[19] \\
\hline \multicolumn{2}{|l|}{ Family Architectonicidae } \\
\hline Adelphotectonica reevei (Hanley, 1862) & 30 \\
\hline Architectonica perspectiva (Linnaeus,1758) & 21,23,24,27,39,41,51,52, [21], A[18],[19],[20] \\
\hline \multicolumn{2}{|l|}{ Family Amathinidae } \\
\hline Amathina tricarinata (Linnaeus, 1767) & $12,14,22$ \\
\hline \multicolumn{2}{|l|}{ Family Pyramidellidae } \\
\hline Longchaeus canaliculatus (Sowerby, 1873) & 50 \\
\hline Otopleura auriscati (Holten,1802) & $\mathrm{A}[19]$ \\
\hline Pyramidella acus (Gmelin, 1791) & $\mathrm{A}[19]$ \\
\hline Pyramidella dolabrata (Muller, 1774) & 51 \\
\hline Pyramidella maculosa Lamarck, 1822 & 38 \\
\hline \multicolumn{2}{|l|}{ Family Cerithiidae } \\
\hline Cerithium citrinumG.B. Sowerby II, 1855 & $\mathrm{~A}[19]$ \\
\hline Cerithium columnaG.B. Sowerby I, 1834 & 51 \\
\hline Cerithium coralium Kiener,1841 & 12,13,14,26-28, [21], A[18],[19],[20] \\
\hline Cerithium echinatum Lamarck, 1822 & $\mathrm{~A}[18]$ \\
\hline Cerithium lifuense Melvill \& Standen, 1895 & 39,52 \\
\hline Cerithium nodulosum Bruguiere,1792 & $\mathrm{A}[18],[19]$ \\
\hline Cerithium novaehollandiaeG.B. Sowerby II, 1855 & $26,46,47,53$ \\
\hline Cerithium torresi Smith,1884 & $3,6,14,17,35,39,46,51,52$ \\
\hline Cerithium trailliiG.B. Sowerby II,1855 & $28,31,35,37,51,55$ \\
\hline Cerithium zonatum (Wood, 1828) & $14,27,29,39,46$ \\
\hline Clypeomorus batillariaeformis Habe \& Kosuge, 1966 & $\mathrm{~A}[18],[20]$ \\
\hline Clypeomorus bifasciata (G.B. Sowerby II,1855) & 26,28,35-37,39, [21],W[17],A[18],[19],[20] \\
\hline Clypeomorus pellucida (Hombron \& Jacquinot,1852) & $14,31,[21], \mathrm{A}[20]$ \\
\hline Clypeomorus petrosa chemnitzianum (Pilsbry, 1901) & 51 \\
\hline Clypeomorus sp. & 46 \\
\hline Rhinoclavis (Rhinoclavis) aspera (Linnaeus,1758) & $6,7,12,13,15-20,27,28,43,51,52,54,55,[21], A[18],[19],[20]$ \\
\hline Rhinoclavis (Rhinoclavis) fasciata (Bruguiere,1792) & [21] \\
\hline Rhinoclavis (Rhinoclavis) sinensis (Gmelin,1791) & $\begin{array}{l}\text { 5,6,9,12-15,17,19,20,26, 27, 29,31,37,39,43,51, } \\
\text { [21],W[17],A[18],[19],[20] }\end{array}$ \\
\hline Rhinoclavis (Procava) sordidula (Gould, 1849) & $38,50,51$ \\
\hline Rhinoclavis (Rhinoclavis) vertagus (Linnaeus,1767) & $7,14,17,44, \mathrm{~A}[19]$ \\
\hline \multicolumn{2}{|l|}{ Family Modulidae } \\
\hline Modulus tectum (Gmelin, 1791) & $51,55, W[17]$ \\
\hline \multicolumn{2}{|l|}{ Family Planaxidae } \\
\hline Planaxis sulcatus (Born,1778) & $\begin{array}{l}2-10,13,15,17-20,26-29,31,35-46,48-52 \text {, } \\
\text { [21],W[17],A[18],[19],[20] }\end{array}$ \\
\hline \multicolumn{2}{|l|}{ Family Potamididae } \\
\hline Cerithidea cingulata (Gmelin,1791) & 31 \\
\hline Telescopium telescopium (Linnaeus,1758) & $34,37, \mathrm{~A}[19]$ \\
\hline
\end{tabular}




\begin{tabular}{|c|c|}
\hline Terebralia palustris (Linnaeus,1767) & 22 \\
\hline \multicolumn{2}{|l|}{ Family Siliquariidae } \\
\hline Tenagodus ponderosus (Mörch, 1861) & $\mathrm{A}[18]$ \\
\hline Tenagodus weldii Tenison-Woods, 1876 & 6,8,9,13,17,19,20,52, W[17],A[18],[20] \\
\hline \multicolumn{2}{|l|}{ Family Turritellidae } \\
\hline Turritella cinguliferaG.B. Sowerby I, 1825 & 52 \\
\hline Turritella terebra (Linnaeus,1758) & 6,21,23,29,39, [21],W[17],A[18],[19],[20] \\
\hline \multicolumn{2}{|l|}{ Family Calyptraeidae } \\
\hline Calyptraea chinensis(Linnaeus,1758) & $\mathrm{A}[19]$ \\
\hline Calyptraea extinctorium Lamarck,1822 & $14,17,21,22,25,30,36,39,51$ \\
\hline Siphopatella walshi (Reeve,1859) & 6,13,22,23,29,30,41,44, W[17],A[19] \\
\hline \multicolumn{2}{|l|}{ Family Cypraeidae } \\
\hline Monetaria annulus (Linnaeus, 1758) & $\mathrm{A}[18],[19],[20]$ \\
\hline Mauritia arabica (Linnaeus, 1758) & $\begin{array}{l}\text { 2-6,9,12,13,15-20,27-29,33,36,37,39,41,44,49,50, } \\
\text { 52,54,55, [21],W[17],A[18],[19],[20] }\end{array}$ \\
\hline Arestorides argus (Linnaeus, 1758) & [21],A[18],[19] \\
\hline Palmadusta asellus (Linnaeus, 1758) & $3,4,13,15,19,27,28,39,40,43,54,[21], \mathrm{W}[17], \mathrm{A}[18],[19],[20$ \\
\hline Monetaria caputserpentis (Linnaeus, 1758) & $\mathrm{A}[18],[19]$ \\
\hline Lyncina carneola (Linnaeus, 1758) & $\mathrm{A}[18],[19]$ \\
\hline Erronea caurica (Linnaeus, 1758) & $\mathrm{W}[17]$ \\
\hline Ovatipsa chinensis (Gmelin, 1791) & 53 \\
\hline Pustularia cicercula (Linnaeus, 1758) & $\mathrm{A}[19]$ \\
\hline Notocypraea comptonii (Gray, 1847) & $5,9,17,19,26,27,29,31,37,43,52,53$ \\
\hline Palmadusta contaminata (G.B. Sowerby I, 1832) & $13,17-20,27,29,41,45,48,52$ \\
\hline Cribrarula cribraria (Linnaeus, 1758) & $9,10,12,29,41,43,52,54, A[18],[19],[20]$ \\
\hline Erronea cylindrica (Born, 1778) & $39,43,44,47-51,53,[21]$ \\
\hline Erosaria erosa (Linnaeus, 1758) & [21], A[18],[19] \\
\hline Erronea errones (Linnaeus, 1758) & $\mathrm{W}[17]$ \\
\hline Purpuradusta gracilis (Gaskoin, 1849) & $12,13,19,20,28,39, \mathrm{~W}[17]$ \\
\hline Purpuradusta hammondae (Iredale, 1939) & 37,55 \\
\hline Bistolida hirundo (Linnaeus, 1758) & $17,19,37,39$ \\
\hline Luria isabella (Linnaeus, 1758) & $\mathrm{A}[19]$ \\
\hline Bistolida kieneri (Hidalgo, 1906) & 27 \\
\hline Erosaria lamarckii (J.E. Gray, 1825) & [21],A[18],[19],[20] \\
\hline Leporicypraea mappa (Linnaeus, 1758) & [21] \\
\hline Mauritia mauritiana (Linnaeus, 1758) & [21],A[19] \\
\hline Erosaria miliaris (Gmelin, 1791) & $8,27,30,37,38,52,55$ \\
\hline Monetaria moneta (Linnaeus, 1758) & $\mathrm{A}[19]$ \\
\hline Nucleolaria nucleus (Linnaeus, 1758) & $\mathrm{A}[19]$ \\
\hline Notocypraea pulicaria (Reeve, 1846) & 19,55 \\
\hline Eclogavena quadrimaculata (J. E. Gray, 1824) & $40,43,52,53, W[17]$ \\
\hline Staphylaea staphylaea (Linnaeus, 1758) & $\mathrm{A}[20]$ \\
\hline
\end{tabular}




\begin{tabular}{|c|c|}
\hline Bistolida stolida (Linnaeus, 1758) & $55, \mathrm{~A}[18]$ \\
\hline Talparia talpa (Linnaeus, 1758) & [21], A[18],[19] \\
\hline Cypraea tigris Linnaeus,1758 & 7,12,13,15,19,20,27-29,35,51, [21],W[17],A[18],[19] \\
\hline Bistolida ursellus (Gmelin, 1791) & 19,40 \\
\hline Lyncina vitellus (Linnaeus, 1758) & $36,38,55,[21]$ \\
\hline Erronea xanthodon (J. E. Gray in G.B. Sowerby I, 1832) & $26,33,37,52$ \\
\hline \multicolumn{2}{|l|}{ Family Ovulidae } \\
\hline Calpurnus verrucosus (Linnaeus, 1758) & $\mathrm{A}[18]$ \\
\hline Crenavolva traillii (A. Adams, 1855) & 20 \\
\hline Cuspivolva tigris (Yamamoto, 1971) & $\mathrm{A}[18]$ \\
\hline $\begin{array}{l}\text { Hiatavolva coarctata (G.B. Sowerby II in A. Adams \& } \\
\text { Reeve, 1848) }\end{array}$ & $\mathrm{A}[18]$ \\
\hline Pellasimnia angasi (Reeve, 1865) & $38,46,47$ \\
\hline Phenacovolva haynesi (Sowerby, 1889) & 41 \\
\hline Phenacovolva rosea (Adams, 1854) & 47, A[18],[19] \\
\hline Phenacovolva lahainaensis (Cate, 1969) & [21],A[18] \\
\hline Prosimnia semperi (Weinkauff, 1881) & $\mathrm{A}[18]$ \\
\hline \multicolumn{2}{|l|}{ Family Littorinidae } \\
\hline Echinolittorina feejeensis (Reeve,1857) & [22] \\
\hline Echinolittorina malaccana(Philippi, 1847) & $\begin{array}{l}2-10,13,15,17-20,26-29,31,36-46,48,49,51,52 \text {, } \\
\text { [21],[22],W[17],A[18],[19],[20] }\end{array}$ \\
\hline Echinolittorina vidua (Gould,1859) & $2,15,17,19,20,27-29,31,36,42,49,[22]$ \\
\hline Littoraria articulata (Philippi, 1846) & 1,32,36,[21],[22],W[17] \\
\hline Littoraria bengalensis Reid, 2001 & $\mathrm{~A}[22]$ \\
\hline Littoraria carinifera (Menke, 1830) & $1,32,[22], \mathrm{A}[20]$ \\
\hline Littoraria conica(Philippi, 1846) & $\mathrm{A}[22]$ \\
\hline Littoraria intermedia (Philippi, 1846) & [22] \\
\hline Littoraria melanostoma (Gray,1839) & $1,32,[21],[22]$ \\
\hline Littoraria pallescens (Philippi, 1846) & $1,32,41,[21],[22]$ \\
\hline Littoraria scabra(Linnaeus,1758) & $\mathrm{A}[18],[19],[20],[22]$ \\
\hline Littoraria strigata (Philippi, 1846) & 1,32, A[18],[19],[20],[22] \\
\hline Littoraria undulata (Gray, 1839) & $\mathrm{A}[20],[22]$ \\
\hline Peasiella roepstorffiana (Nevill, 1885) & 2,15,19, [22],A[20] \\
\hline \multicolumn{2}{|l|}{ Family Naticidae } \\
\hline Eunaticina papilla (Gmelin,1791) & $8,10,22,29$ \\
\hline Euspira yokoyamai (Kuroda \& Habe, 1952) & 26 \\
\hline Natica arachnoidea (Gmelin,1791) & 27 \\
\hline Stigmaulax cayennensis (Récluz, 1850) & 51 \\
\hline Naticarius colliei (Récluz, 1844) & 39 \\
\hline Tanea euzona (Récluz, 1844) & 53 \\
\hline Natica fasciata (Roding,1798) & 50 \\
\hline Notocochlis gualteriana (Récluz, 1844) & $12,19,20,29,31,52$ \\
\hline
\end{tabular}




\begin{tabular}{|c|c|}
\hline Tanea lineata (Röding, 1798) & $18,23,41,52,55, \mathrm{~A}[19]$ \\
\hline Natica luculenta Iredale, 1929 & 29,55 \\
\hline Naticarius onca (Röding, 1798) & $16,18, A[18],[20]$ \\
\hline Polinices candidissimus (Le Guillou, 1842) & 3 \\
\hline Natica schepmani Thiele, 1925 & 53 \\
\hline Natica stellata Hedley, 1913 & $19,25,51$ \\
\hline Notocochlis tigrina (Röding, 1798) & [21],A[19],[20] \\
\hline Tanea undulata (Röding, 1798) & 51 \\
\hline Natica unifasciata Lamarck, 1822 & 19,20 \\
\hline Natica vitellus (Linnaeus,1758) & $21,23,24,27,31,41,49,51,52,55, W[17]$ \\
\hline Tanea zelandica (Quoy \& Gaimard, 1832) & $28,37,52$ \\
\hline Polinices albumen (Linnaeus, 1758) & [21] \\
\hline Neverita didyma (Röding, 1798) & $13,21,25,[21], \mathrm{A}[19]$ \\
\hline Mammilla mammata (Röding, 1798) & $33,35-37,51,55$ \\
\hline Polinices mammilla (Linnaeus, 1758) & $\begin{array}{l}\text { 2-9,11-15,17-20,25-29,38,39,41,42,44,46-55 } \\
\text { [21],W[17],A[18],[19],[20] }\end{array}$ \\
\hline Polinices powisianus (Récluz, 1844) & 10,38 \\
\hline Polinices putealis Garrard,1961 & 14,26,27, \\
\hline Mammilla sebae (Récluz, 1844) & $\mathrm{A}[18],[19]$ \\
\hline Mammilla simiae (Deshayes, 1838) & $\mathrm{A}[20]$ \\
\hline Sigatica bathyraphe (Pilsbry, 1911) & 9 \\
\hline Sinum haliotoideum (Linnaeus, 1758) & 27 \\
\hline Sinum incisum (Reeve, 1864) & $\mathrm{A}[18]$ \\
\hline \multicolumn{2}{|l|}{ Family Strombidae } \\
\hline Lambis lambis (Linnaeus,1758) & 51,[21], A[18],[19] \\
\hline Harpago chiragra (Linnaeus, 1758) & [21],A[18],[19] \\
\hline Lambis scorpius (Linnaeus,1758) & $\mathrm{A}[18],[19]$ \\
\hline Lambis truncata([Lightfoot], 1786) & $\mathrm{A}[18],[19]$ \\
\hline Laevistrombus canarium (Linnaeus, 1758) & $\begin{array}{l}9,11,14,21,22,24,25,28,33,36,37,39,41,50,[21], W[17 \\
\text {,A[19],[20] }\end{array}$ \\
\hline Canarium erythrinum (Dillwyn, 1817) & 46 \\
\hline Canarium labiatum (Röding, 1798) & 18,55, A[19],[20] \\
\hline Lentigo lentiginosus (Linnaeus, 1758) & $\mathrm{A}[18]$ \\
\hline Conomurex luhuanus (Linnaeus, 1758) & $\begin{array}{l}9,15,17,20,28,29,37,41,43,44,51,52,54,55 \\
\text { [21],A[18],[19],[20] }\end{array}$ \\
\hline Margistrombus marginatus (Linnaeus, 1758) & 13,26, W[17],A[18],[19],[20] \\
\hline Margistrombus robustus (G.B. Sowerby II, 1875) & $18,21,23,25,51$ \\
\hline Margistrombus septimus (Duclos, 1844) & 31 \\
\hline Dolomena plicata (Röding, 1798) & 52 \\
\hline Dolomena pulchella (Reeve, 1851) & 29 \\
\hline Strombus sp. & 26 \\
\hline Terestrombus terebellatus (G.B. Sowerby II, 1842) & $\mathrm{A}[19]$ \\
\hline Canarium urceus (Linnaeus, 1758) & 8,14-20,27-29,37,39,41,50-53,55, [21],W[17],A[18],[19] \\
\hline
\end{tabular}




\begin{tabular}{|c|c|}
\hline Dolomena variabilis (Swainson, 1820) & $14,27,30,51$ \\
\hline Doxander vittatus apicatus (Man in ‘t Veld \& Visser, 1993) & $21,23,39,53,55$ \\
\hline Doxander vittatus vittatus (Linnaeus, 1758) & 23,27,29,31,37,51, [21],W[17],A[19] \\
\hline Canarium wilsonorum (Abbott, 1967) & $27,41,44$ \\
\hline Terebellum terebellum (Linnaeus,1758) & 55 \\
\hline \multicolumn{2}{|l|}{ Family Bursidae } \\
\hline Bufonaria rana (Linnaeus,1758) & 6,7,21,24,29,38,39,44, [21],W[17] \\
\hline \multicolumn{2}{|l|}{ Family Cassidae } \\
\hline Casmaria ponderosa (Gmelin, 1791) & $\mathrm{A}[18]$ \\
\hline Cassis cornuta (Linnaeus,1758) & $47,[21]$ \\
\hline Phalium areola(Linnaeus,1758) & $\mathrm{A}[19]$ \\
\hline Phalium glaucum (Linnaeus,1758) & 8,18,21,24,38, [21],A[18],[19],[20] \\
\hline Semicassis bisulcata (Schubert\&Wagner,1829) & 6,15,26,29,31,38,39,44,52,54,55.W[17], A[19],[20] \\
\hline \multicolumn{2}{|l|}{ Family Tonnidae } \\
\hline Tonna dolium (Linnaeus,1758) & $\mathrm{A}[19]$ \\
\hline \multicolumn{2}{|l|}{ Family Ranellidae } \\
\hline Lotoria perryi (Emerson \& Old, 1963) & $\mathrm{A}[18]$ \\
\hline Monoplex pilearis (Linnaeus, 1758) & $\mathrm{A}[18],[19]$ \\
\hline Ranularia oboesa (Perry, 1811) & $\mathrm{A}[18]$ \\
\hline Gyrineum bituberculare (Lamarck, 1816) & 50 \\
\hline Gyrineum gyrinum (Linnaeus, 1758) & 27,51, A[18],[19] \\
\hline Gyrineum natator (Roding,1798) & $31, \mathrm{~A}[18]$ \\
\hline Septa rubecula (Linnaeus, 1758) & $\mathrm{A}[18]$ \\
\hline \multicolumn{2}{|l|}{ Family Personidae } \\
\hline Distorsio reticularis (Linnaeus, 1758) & $\mathrm{A}[18]$ \\
\hline \multicolumn{2}{|l|}{ Family Ficidae } \\
\hline Ficus gracilis (G.B. Sowerby I, 1825) & $\mathrm{A}[20]$ \\
\hline Ficus variegata Roding,1798 & $23,30,31$ \\
\hline \multicolumn{2}{|l|}{ Family Hipponicidae } \\
\hline Cheilea equestris (Linnaeus, 1758) & $\mathrm{A}[19]$ \\
\hline Cheilea hipponiciformis (Reeve, 1858) & 10,18 \\
\hline \multicolumn{2}{|l|}{ Family Vanikoridae } \\
\hline Vanikoro fenestrata (A.Adams, 1863) & $9,12,13,20,50,55$ \\
\hline Vanikoroplicata(Recluz, 1844) & $13, \mathrm{~A}[18]$ \\
\hline Vanikoro helicoidea(Guillu, 1842) & W[17], A[19] \\
\hline \multicolumn{2}{|l|}{ Family Triviidae } \\
\hline Trivirostra oryza (Lamarck, 1810) & $\mathrm{A}[19],[20]$ \\
\hline Trivirostra pellucidula (Gaskoin,1846) & 43,55 \\
\hline \multicolumn{2}{|l|}{ Family Velutinidae } \\
\hline CoriocellahibyaeWellens, 1991 & $\mathrm{~A}[18],[20]$ \\
\hline Coriocella nigra Blainville, 1824 & $\mathrm{~A}[18],[20]$ \\
\hline
\end{tabular}




\begin{tabular}{|c|c|}
\hline Family Vermetidae & \\
\hline Thylacodes medusae Pilsbry, 1891 & 25,A[18] \\
\hline Family Xenophoridae & \\
\hline Xenophora (Xenophora) solarioides (Reeve, 1845) & 51 \\
\hline Family Cancellariidae & \\
\hline Trigonostoma bicolor (Hinds, 1843) & 23 \\
\hline Family Conidae & \\
\hline Conus arenatus Hwass in Bruguière, 1792 & 50 \\
\hline Conus aulicus Linnaeus, 1758 & $\mathrm{~A}[18],[19]$ \\
\hline Conus betulinus Linnaeus, 1758 & 44, A[18],[20] \\
\hline Conus capitaneus Linnaeus, 1758 & $43,48,52$ \\
\hline Conus caracteristicus Fischer von Waldheim,1807 & 28,39,41,51,52,55,[21],A[18] \\
\hline Conus daucus daucus Hwass in Bruguière, 1792 & $\mathrm{~A}[19]$ \\
\hline Conus cocceus Reeve, 1844 & 43 \\
\hline Conus coelinae Crosse, 1858 & 41 \\
\hline Conus spectrum Linnaeus, 1758 & 5 \\
\hline Conus coronatus Gmelin, 1791 & $27,39,51, \mathrm{~A}[20]$ \\
\hline Conus cumingii Reeve,1848 & 31 \\
\hline Conus cyanostoma Adams, 1855 & 41 \\
\hline Conus distans Hwass in Bruguiere, 1792 & $\mathrm{~A}[19]$ \\
\hline Conus eburneus Hwass in Bruguière, 1792 & [21],A[18],[19] \\
\hline Conus emaciatus Reeve, 1849 & $\mathrm{~A}[18]$ \\
\hline Conus eximius Reeve, 1849 & $41,46,50,52,55$ \\
\hline Conus figulinus Linnaeus, 1758 & {$[21]$} \\
\hline Conus flavidus Lamarck, 1810 & $\mathrm{~A}[18]$ \\
\hline Conus generalis Linnaeus, 1767 & [21], A[18] \\
\hline Conus glaucus Linnaeus, 1758 & 31 \\
\hline Conus grangeriG.B. Sowerby III, 1900 & $41,46,53$ \\
\hline Conus legatus Lamarck, 1810 & 9 \\
\hline Conus litteratus Linnaeus, 1758 & 38,41,44,52, [21],A[18],[19] \\
\hline Conus lividus Hwass in Bruguière, 1792 & $\mathrm{~A}[19]$ \\
\hline Conus magus Linnaeus, 1758 & $\mathrm{~A}[19]$ \\
\hline Conus marmoreus Linnaeus,1758 & 17 \\
\hline Conus miles Linnaeus, 1758 & $\mathrm{~A}[18],[19]$ \\
\hline Conus miliaris Hwass in Bruguiere, 1792 & $29,46,54,[21]$ \\
\hline Conus monachus Linnaeus,1758 & $29,31,36,46$ \\
\hline Conus achatinus Gmelin, 1791 & {$[21]$} \\
\hline Conus mustelinus Hwass in Bruguière, 1792 & $\mathrm{~W}[17], \mathrm{A}[19]$ \\
\hline Conus nussatella Linnaeus, 1758 & $\mathrm{~A}[19]$ \\
\hline Conus pennaceus Born, 1778 & $\mathrm{~A}[18]$ \\
\hline Conus pulcher[Lightfoot],1786 & 27 \\
\hline Conus quercinus Lightfoot, 1786 & 39,41 \\
\hline
\end{tabular}




\begin{tabular}{|c|c|}
\hline Conus rufimaculosus Macpherson, 1959 & $29,41,42,46,52,55$ \\
\hline Conus striatus Linnaeus, 1758 & $\mathrm{~A}[20]$ \\
\hline Conus striolatus Kiener, 1848 & $20,48,52$ \\
\hline Conus terebra Born,1778 & 28,48 \\
\hline Conus tessulatus Born,1778 & $41,52,55,[21]$ \\
\hline Conus textile Linnaeus,1758 & $9,10,19,36,38,54,[21]$ \\
\hline Conus vexillum Gmelin, 1791 & 39,41,[21], A[18],[19] \\
\hline Conus victoriae Reeve, 1843 & $29,39,40$ \\
\hline Conus nodulosusG.B. Sowerby II, 1864 & 55 \\
\hline Conus virgo Linnaeus, 1758 & 55, [21], A[18],[19] \\
\hline Conus planorbis Born, 1778 & 47 \\
\hline Conus zonatus Hwass in Bruguiere, 1792 & $\mathrm{~A}[18]$ \\
\hline \multicolumn{2}{|l|}{ Family Terebridae } \\
\hline Terebra babylonia Lamarck, 1822 & $\mathrm{~A}[18],[20]$ \\
\hline Cinguloterebra boucheti (Bratcher, 1981) & 26 \\
\hline Cinguloterebra caddeyi (Bratcher \& Cernohorsky, 1982) & 41 \\
\hline Terebra cingulifera Lamarck, 1822 & 10,18 \\
\hline Terebra laevigata Gray, 1834 & 9 \\
\hline Oxymeris maculata (Linnaeus, 1758) & $43,50,55,[21]$ \\
\hline Strioterebrum nitidum (Hinds, 1844) & 26 \\
\hline Terebra quoygaimardi Cernohorsky \& Bratcher, 1976 & 26 \\
\hline Terebra subulata (Linnaeus, 1767) & $\mathrm{A}[19]$ \\
\hline Terebra textilis Hinds, 1844 & 50 \\
\hline Myurella undulata (Gray, 1834) & 17,18 \\
\hline \multicolumn{2}{|l|}{ Family Drilliidae } \\
\hline Clavus exasperatus (Reeve, 1843) & 51 \\
\hline Cymatosyrinx impolita Kuroda, Habe \& Oyama, 1971 & 50 \\
\hline \multicolumn{2}{|l|}{ Family Turridae } \\
\hline Unedogemmula unedo (Kiener, 1839) & 38 \\
\hline Ptychobela flavidula (Lamarck, 1822) & 38 \\
\hline \multicolumn{2}{|l|}{ Family Clavatulidae } \\
\hline Turricula javana (Linnaeus,1758) & $1,21,22,31$ \\
\hline \multicolumn{2}{|l|}{ Family Babyloniidae } \\
\hline Babylonia areolata (Link,1807) & 21,31 \\
\hline Babylonia spirata (Linnaeus,1758) & $\mathrm{A}[19]$ \\
\hline \multicolumn{2}{|l|}{ Family Buccinidae } \\
\hline Pollia bednalli (G.B.Sowerby, 1895) & 23 \\
\hline Pollia fumosa (Dillwyn, 1817) & $9,10,17,20,31,46,50-52,55, \mathrm{~W}[17], \mathrm{A}[19]$ \\
\hline Pollia undosa (Linnaeus, 1758) & $9,13,17,19,20,39,[21], A[19]$ \\
\hline Engina armillata (Reeve, 1846) & 16 \\
\hline Engina mendicaria (Linnaeus,1758) & $\mathrm{A}[19],[20]$ \\
\hline Nassaria pusilla Roding, 1798 & $\mathrm{~W}[17]$ \\
\hline
\end{tabular}




\begin{tabular}{|c|c|}
\hline Phos sculptilis Wattson, 1886 & 41 \\
\hline Phos senticosus (Linnaeus,1758) & $13,21,23,26,27,37,41$ \\
\hline Pisania ignea (Gmelin, 1791) & $\mathrm{A}[18]$ \\
\hline Clivipollia pulchra (Reeve, 1846) & $\mathrm{A}[20]$ \\
\hline Solenosteira gatesi Berry, 1963 & 25 \\
\hline \multicolumn{2}{|l|}{ Family Colubrariidae } \\
\hline Colubraria muricata (Lightfoot,1786) & $\mathrm{A}[18]$ \\
\hline \multicolumn{2}{|l|}{ Family Melongenidae } \\
\hline Hemifusus ternatanus (Gmelin,1791) & $1,18,21,22,24,32,39,44,52, \mathrm{~W}[17]$ \\
\hline Pugilina cochlidium (Linnaeus,1758) & $1,12,14,17,21,22,28, A[18],[20]$ \\
\hline \multicolumn{2}{|l|}{ Family Nassariidae } \\
\hline Ilyanassa obsoleta (Say,1822) & 31 \\
\hline Nassarius castus (Gould,1850) & 37 \\
\hline Nassarius clarus (Marrat, 1877) & $6,8,10,17,18,30,51,52$ \\
\hline Nassarius comptus (A. Adams, 1852) & $11,15,38,39,51,52$ \\
\hline Nassarius concinnus (Powys, 1835) & 23,31 \\
\hline Nassarius crematus (Hinds,1844) & $18,23,26,27,31,39,41,46,[21]$ \\
\hline Nassarius distortus (A. Adams, 1852) & 28,29,38,46,50-52,A[19] \\
\hline Nassarius dorsatus (Roding,1798) & 13,46,55,[21],A[18],[20] \\
\hline Nassarius gaudiosus (Hinds,1844) & 2 \\
\hline Nassarius globosus (Quoy \& Gaimard, 1833) & [21],A[18],[19],[20] \\
\hline Nassarius jacksonianus(Quoy \& Gaimard, 1833) & $\mathrm{A}[18],[19],[20]$ \\
\hline Nassarius livescens (Philippi, 1849) & $\mathrm{W}[17], \mathrm{A}[20]$ \\
\hline Nassarius margaritifer (Dunker, 1847) & $25,[21]$ \\
\hline Nassarius pullus (Linnaeus, 1758) & 22,[21],A[18] \\
\hline Nassarius nodifer (Powys, 1835) & 9 \\
\hline \multicolumn{2}{|l|}{ Family Columbellidae } \\
\hline Anachis sanfelipensis Lowe,1935 & 26 \\
\hline Euplica turturina (Lamarck, 1822) & $\mathrm{A}[18]$ \\
\hline Euplica bidentata (Menke, 1843) & 14,43 \\
\hline Pyrene flava (Bruguiere,1789) & 12 \\
\hline Pyrene punctata (Bruguiere,1789) & $9,12,13,16,17,19,20,51, A[18]$ \\
\hline Euplica scripta (Lamarck,1822) & $12,13,15,17,31,39,46,47,51,[21], W[17], A[18],[19],[20]$ \\
\hline Pyrene testudinaria (Link, 1807) & $\mathrm{W}[17]$ \\
\hline \multicolumn{2}{|l|}{ Family Costellariidae } \\
\hline Vexillum (Costellaria) radix (G. B. Sowerby II, 1874) & 39,52 \\
\hline Vexillum (Costellaria) obeliscus (Reeve, 1844) & 51 \\
\hline Vexillum (Vexillum) plicarium (Linnaeus, 1758) & $\mathrm{A}[19]$ \\
\hline Vexillum sp. & 38 \\
\hline \multicolumn{2}{|l|}{ Family Harpidae } \\
\hline Harpa articularis Lamarck,1822 & $24,39,52,[21]$ \\
\hline Harpa major (Roding,1798) & $\mathrm{A}[18]$ \\
\hline
\end{tabular}




\begin{tabular}{|c|c|}
\hline Family Marginellidae & \\
\hline Cryptospira dactylus (Lamarck, 1822) & 27 \\
\hline Cryptospira elegans (Gmelin, 1791) & $\mathrm{A}[20]$ \\
\hline Cryptospira praecallosa (Higgins, 1876) & $\mathrm{A}[19]$ \\
\hline Cryptospira tricincta (Hinds, 1844) & 11,27 \\
\hline \multicolumn{2}{|l|}{ Family Mitridae } \\
\hline Cancilla aegra (Reeve, 1845) & 9 \\
\hline Domiporta granatina (Lamarck, 1811) & 51 \\
\hline Domiporta praestantissima (Roding,1798) & 37 \\
\hline Mitra ambigua Swainson,1829 & 36 \\
\hline Mitra aurantia (Gmelin,1791) & $\begin{array}{l}\text { 5, 8,9,11-13,15-20,26-29, 31,33, 37, 39, 42, } 51 \\
\text { [21],W[17],A[19],[20] }\end{array}$ \\
\hline Mitra chrysalis Reeve, 1844 & 42 \\
\hline Mitra colombelliformisKiener, 1838 & $\mathrm{~A}[19]$ \\
\hline Mitra contractaSwainson, 1820 & $\mathrm{~A}[19]$ \\
\hline Mitra fraga Quoy \& Gaimard,1833 & 26 \\
\hline Mitra paupercula(Linnaeus, 1758) & $\mathrm{A}[20]$ \\
\hline Mitra scutulata (Gmelin, 1791) & [21],A[19] \\
\hline Mitra tabanula Lamarck, 1811 & $27-29$ \\
\hline Mitra ustulata Reeve, 1844 & $\mathrm{~A}[18]$ \\
\hline Neocancilla circula (Keiner, 1838) & 27 \\
\hline Scabricola fissurata (Lamarck,1811) & 38,55 \\
\hline Scabricola ocellata (Swainson, 1831) & 18,38 \\
\hline Ziba flammea (Quoy \& Gaimard, 1833) & $38,39,55$ \\
\hline Subcancilla interlirata (Reeve, 1844) & $41,52,[21]$ \\
\hline \multicolumn{2}{|l|}{ Family Muricidae } \\
\hline Chicoreus brunneus (Link,1807) & $\begin{array}{l}4,7,9,10,16,17,19,20,25-27,29,36,39,48,51,52,55 \\
\text { [21],W[17],A[18],[19],[20] }\end{array}$ \\
\hline Chicoreus capucinus (Lamarck,1822) & 1,31, A[18],[19],[20] \\
\hline Chicoreus ramosus (Linnaeus,1758) & 7, [21],A[18],[19],[20] \\
\hline Chicoreus torrefactus (G. B. Sowerby II, 1841) & 9,15,19,27,37,43, [21],A[18],[19],[20] \\
\hline Coralliophila fearnleyi (Emerson \& D’Attilio, 1965) & [21] \\
\hline Morula (Habromorula) biconica (Blainville, 1832) & [21],W[17], A,[18],[20] \\
\hline Ergalatax contracta (Reeve, 1846) & $2,3,13,16,50,51$ \\
\hline $\begin{array}{l}\text { Drupella margariticola (Broderip, in Broderip \& Sowerby, } \\
\text { 1833) }\end{array}$ & 5,12-14,16,17,19,20,27,29,37,39, [21],W[17], A,[18],[20] \\
\hline Drupella rugosa (Born,1778) & 8,12,15-17,19,20,26-29,33,37,39,40,43,55,W[17] \\
\hline Homalocantha scorpio (Linnaeus, 1758) & $\mathrm{A}[18]$ \\
\hline Homalocantha zamboi (Burch \& Burch, 1960) & $\mathrm{A}[18]$ \\
\hline Indothais malayensis (Tan \& Sigurdsson, 1996) & 25 \\
\hline Lataxiena blosvillei (Deshayes, 1832) & 21 \\
\hline Orania ficula (Reeve, 1848) & 2,4,6,7,9,10,14,15,26,37,39 \\
\hline Lataxiena fimbriata (Hinds, 1844) & 46 \\
\hline
\end{tabular}




\begin{tabular}{|c|c|}
\hline Morula (Morula) anaxares (Kiener, 1836) & $\mathrm{A}[20]$ \\
\hline Tenguella granulata (Duclos, 1832) & [21],A[19] \\
\hline Tenguella marginalba (Blainville, 1832) & $\mathrm{A}[20]$ \\
\hline Tenguella musiva (Kiener, 1835) & $19,20,28,29,31,37,39,46,51,55,[21], W[17], A[20]$ \\
\hline Semiricinula squamosa (Pease, 1868) & 20 \\
\hline $\begin{array}{l}\text { Morula (Habromorula) spinosa (H. Adams \& A. Adams, } \\
\text { 1853) }\end{array}$ & $6,13,15-19,26,29,33,39,42,43,51,55$ \\
\hline Murex djarianensis poppei Houart, 1979 & [21],A,[18],[19],[20] \\
\hline Murex scolopax Dillwyn, 1817 & $\mathrm{~A}[18]$ \\
\hline Murex (Murex) trapa Röding, 1798 & $15,17,22,24,29,31,37,39,[21], W[17], A[18],[19],[20]$ \\
\hline Murex (Murex) tribulus Linnaeus, 1758 & 27,45 \\
\hline Murex troscheli troscheli Lischke,1868 & 38,A[18] \\
\hline Pseudoneptunea varicosa (Gmelin,1791) & 34 \\
\hline Rapana rapiformis (Born,1778) & 24,31 \\
\hline Thalessa aculeata (Deshayes \& Milne-Edwards, 1844) & $9,11,13,15,19,20,25,28,29,31,[21], W[17], A[18],[19],[20]$ \\
\hline Mancinella alouina (Röding, 1798) & $29,[21]$ \\
\hline Reishia bitubercularis (Lamarck, 1822) & 13,25,31, [21],A[18],[19],[20] \\
\hline Reishia bronni (Dunker, 1860) & $\mathrm{A}[18]$ \\
\hline Mancinella echinata (Blainville, 1832) & $\begin{array}{l}2-10,12,14-19,26-29,33,36,37,46-48,52,55 \\
\text { W[17],A[18],[20] }\end{array}$ \\
\hline Mancinella echinulata (Lamarck, 1822) & $\mathrm{A}[20]$ \\
\hline Indothais lacera (Born, 1778) & 51 \\
\hline Mancinella alouina (Röding, 1798) & $\mathrm{A}[18],[20]$ \\
\hline Semiricinula muricina (Blainville, 1832) & $\mathrm{W}[17], \mathrm{A}[20]$ \\
\hline Indothais rufotincta (K. S. Tan \& Sigurdsson, 1996) & 31 \\
\hline Indothais sacellum (Gmelin, 1791) & 31 \\
\hline Menathais tuberosa (Röding, 1798) & [21],A[20] \\
\hline \multicolumn{2}{|l|}{ Family Fasciolariidae } \\
\hline Benimakia lanceolata (Reeve, 1847) & $\mathrm{A}[18]$ \\
\hline Filifusus filamentosus (Röding, 1798) & $\mathrm{A}[18],[19],[20]$ \\
\hline Pleuroploca trapezium (Linnaeus, 1758) & $\mathrm{A}[18],[19],[20]$ \\
\hline \multicolumn{2}{|l|}{ Family Olividae } \\
\hline Oliva annulata (Gmelin, 1791) & $19,42,53,55$ \\
\hline Oliva hirasei Kira,1959 & 26,27 \\
\hline Agaronia plicaria Lamarck, 1811 & 41 \\
\hline Oliva miniacea Roding, 1798 & $21,37,41,50,52$ \\
\hline Oliva oliva (Linnaeus,1758) & $24,27,29,37,39,51,55,[21]$ \\
\hline Agaronia gibbosa (Born, 1778) & $\mathrm{A}[19]$ \\
\hline \multicolumn{2}{|l|}{ Family Turbinellidae } \\
\hline Vasum turbinellus (Linnaeus,1758) & 19,39,48,52,55, [21],W[17],A[18],[19],[20] \\
\hline \multicolumn{2}{|l|}{ Family Volutidae } \\
\hline Cymbiola nobilis (Lightfoot,1786) & 7,21,24,27,29,51,[21] \\
\hline Melo melo (Lightfoot,1786) & 7,31, [21],W[17],A[19] \\
\hline
\end{tabular}




\begin{tabular}{|c|c|}
\hline \multicolumn{2}{|l|}{ Family Janthinidae } \\
\hline Janthina globosa Swainson,1822 & $27,51,52,55,[21]$ \\
\hline \multicolumn{2}{|l|}{ Family Eulimidae } \\
\hline Melanellabovicornu(Pilsbry, 1905) & $\mathrm{A}[18]$ \\
\hline Thyca crystallina (Gould, 1846) & $\mathrm{A}[19]$ \\
\hline \multicolumn{2}{|l|}{ Family Epitoniidae } \\
\hline Epidendrium billeeanum (DuShane \& Bratcher, 1965) & $\mathrm{A}[18]$ \\
\hline \multicolumn{2}{|l|}{ Family Acteonidae } \\
\hline Pupa nitidula (Lamarck, 1816) & $\mathrm{W}[17\}$ \\
\hline Pupa solidula (Linnaeus,1758) & 2,26 \\
\hline Pupa solidula fumata (Reeve, 1865) & 51 \\
\hline Pupa strigosa (Gould, 1859) & 18 \\
\hline Pupa sulcata (Gmelin, 1791) & 17 \\
\hline Pupa sp.1 & 20 \\
\hline Pupa sp.2 & 9 \\
\hline \multicolumn{2}{|l|}{ Family Haminoeidae } \\
\hline Aliculastrum cylindricum (Helbling, 1779) & $17,18,38,39,41-45,48,50-52,55$ \\
\hline Atys naucum (Linnaeus,1758) & $5,13,17,18,23,31,39,46,51-53, A[19]$ \\
\hline Liloa porcellana (Gould, 1859) & 14 \\
\hline \multicolumn{2}{|l|}{ Family Bullidae } \\
\hline Bulla ampulla Linnaeus, 1758 & [21],A[18],[19],[20] \\
\hline Bulla striata Bruguiere,1792 & $\mathrm{A}[20]$ \\
\hline \multicolumn{2}{|l|}{ Family Bullinidae } \\
\hline Bullina lineata (Gray, 1825) & $\mathrm{A}[18]$ \\
\hline \multicolumn{2}{|l|}{ Subclass PULMONATA } \\
\hline \multicolumn{2}{|l|}{ Family Siphonariidae } \\
\hline Siphonaria atra Quoy \& Gaimard, 1833 & 28,29,39, W[17],A[18],[20] \\
\hline Siphonaria laciniosa (Linnaeus, 1758) & $\mathrm{A}[18],[20]$ \\
\hline Siphonaria subatra Pilsby, 1904 & $28,29,39$ \\
\hline \multicolumn{2}{|l|}{ Family Ellobiidae } \\
\hline Cassidula aurisfelis (Bruguiere,1789) & $32, \mathrm{~A}[20]$ \\
\hline Ellobium aurisjudae (Linnaeus,1758) & 21,34 \\
\hline Ellobium aurismidae(Linnaeus,1758) & 34 \\
\hline Pythia reeveana Pfeiffer, 1853 & $\mathrm{~A}[20]$ \\
\hline
\end{tabular}

